\title{
Numerical simulation as a modeling and teaching tool of optical devices and systems
}

Tony Yuk, Siu Fung Yu, Ping Shum, Joseph Palais

Tony T. Yuk, Siu Fung Yu, Ping Shum, Joseph C. Palais, "Numerical simulation as a modeling and teaching tool of optical devices and systems," Proc. SPIE 3190, Fifth International Topical Meeting on Education and Training in Optics, (8 December 1997); doi: 10.1117/12.294393

Event: Fifth International Topical Meeting on Education and Training in Optics, 1997, Delft, Netherlands 


\title{
Numerical Simulation as a Modeling and Teaching Tool of Optical Devices and Systems
}

\author{
T.I. Yuk, S.F. Yu, P. Shum, and J.C. Palais* \\ Department of Electrical \& Electronic Engineering, University of Hong Kong, Pokfulam, Hong Kong \\ Email: tiyuk@eee.hku.hk \\ *Department of Electrical Engineering, Arizona State University, Tempe, AZ.
}

\begin{abstract}
This paper describes the development of numerical simulation models of an Er-doped waveguide laser and a mode-locked fiber soliton laser. The Er-doped waveguide laser model is a simple and straight-forward but powerful dynamic model using time domain algorithm. It is based on i) time dependent rate equations of a quasi-two-level-system for the population densities and ii) time-dependent traveling wave equations for the pump and signal power which are solved simultaneously in time-domain. The dynamic responses of population densities, pump and signal power are investigated. The model is used to study more sophisticated structure with cross-coupling from optical feedback of an etched grating. Another simulation model is developed to investigate the generation of sub-picosecond solitons in an active mode-locked fiber ring laser which consists of a polarization preserving Er-doped single mode fiber, an amplitude modulator and a phase modulator and has taken into account of dispersive spreading, self-phase modulation, finite amplification bandwidth, pump depletion, and Raman self-frequency shift. A newly developed numerical technique, Fourier Series Analysis Technique, is used to solve the non-linear Schrodinger equation of soliton propagation. Time trace of the soliton pulse propagation and its spectrum can be obtained under a wide range of operation conditions.
\end{abstract}

Keywords : simulation models, waveguide laser, fiber laser, solitons

\section{INTRODUCTION}

Wide spread research and development efforts are being spent in the field of opto-electronics. Numerous passive and active optical devices are designed and developed. Analytic and numerical modeling are the tools for designing and optimizing these devices and systems. For more advanced devices and systems, numerical simulation is often a better tool that can take into account more complex structures and configurations. Effects of various device and system parameters can be systematically studied in greater detail and compared with experimental data. In this paper, we describe numerical simulation models of an Er-doped waveguide laser and a mode-locked fiber soliton laser. The simulation models have been assigned and developed as student design projects. Understanding of these laser devices are greatly enhanced through the development of the simulation models.

Several numerical models have been recently developed to analyze the relaxation oscillation behavior of Erdoped Ti:LiNbO ${ }_{3}$ waveguide laser [1,2]. In this paper we present a more straight-forward time-domain algorithm to calculate the dynamic response based on directly solving the time-dependent wave equations and the time-dependent quasi-two-level-laser rate equations. The model take into consideration of the longitudinal distribution of spontaneous emission noise. The transient responses of population densities, pump and signal power are investigated for Er3+-doped Ti:LiNbO3 waveguide lasers. To demonstrate the sophistication of this time-domain model, the influence of crosscoupled effect of the traveling waves due to the optical feedback of an etched grating is also studied. Dynamic response of the waveguide laser with and without grating are compared.

A second numerical simulation model is developed to investigate the generation of sub-picosecond solitons in active mode-locked fiber ring laser in both time and frequency domains. The laser model consists of a polarization preserving Er-doped single mode fiber, an amplitude modulator and a phase modulator. A relatively straight forward method is used to model the fiber laser using Fourier Series Analysis Technique. The advantages of using FSAT rather than the conventional split-step Fourier Method include better computation efficiency, less number of sampling points, and can handle higher order nonlinear terms without resort to other numerical methods. The model has taken into account of dispersive spreading, self-phase modulation, finite amplification bandwidth, pump depletion, and Raman self- 
frequency shift. Time trace of the soliton pulse propagation and its spectrum can be obtained under a wide range of operation conditions.

\section{MODELING OF Er ${ }^{3+}$-DOPED WAVEGUIDE LASER}

\subsection{Theoretical model}

The schematic of optical pumped waveguide laser is shown in figure 1. $\mathrm{The}^{3+}$ ion is assumed to be uniformly distributed and have a concentration $N_{t}$. A grating is etched along the $\mathrm{z}$ direction to provide optical feedback. In our model, light of orthogonal $(\sigma-)$ polarization (to the optical axis) is only considered in our analysis.

The interaction of the pump/signal photons with the $\mathrm{Er}^{3+}$ ions can be described by the rate equations of the populations $N_{2}$ and $N_{1}$ for the upper and lower levels expressed below [1], [2]:

$$
\begin{aligned}
& \frac{d N_{2}}{d t}=\left(R_{12}+W_{12}\right) N_{1}-\left(A_{21}+R_{21}+W_{21}\right) N_{2} \\
& N_{1}=N_{t}-N_{2}
\end{aligned}
$$

where $R_{12}\left(W_{12}\right)$ and $R_{21}\left(W_{21}\right)$ are the pump (signal) absorption and emission rates respectively, $A_{21}=1 / \tau$ is the spontaneous rate of transition from the excited state to the ground state.

The evolution of the forward and backward propagating pump(signal) fields $\mathrm{p}^{+}\left(\mathrm{s}^{+}\right)$and $\mathrm{p}^{-}\left(\mathrm{s}^{-}\right)$is based on the time dependent travelling wave equation [3], [4]:

$$
\begin{aligned}
& \left( \pm \frac{\partial}{\partial \mathrm{z}}+\frac{1}{\mathrm{c}_{\mathrm{n}}} \frac{\partial}{\partial \mathrm{t}}\right) \mathrm{p}^{ \pm}=\frac{1}{2}\left(-\bar{\alpha}_{\mathrm{P}}+\sigma_{\mathrm{P}}^{21} \mathrm{~N}_{2}-\sigma_{\mathrm{P}}^{12} \mathrm{~N}_{1}\right) \mathrm{p}^{ \pm}+i \kappa \mathrm{p}^{\mp}+\tilde{\chi}_{\mathrm{P}} \\
& \left( \pm \frac{\partial}{\partial \mathrm{z}}+\frac{1}{\mathrm{c}_{\mathrm{n}}} \frac{\partial}{\partial \mathrm{t}}\right) \mathrm{s}^{ \pm}=\frac{1}{2}\left(-\bar{\alpha}_{\mathrm{S}}+\sigma_{\mathrm{S}}^{21} \mathrm{~N}_{2}-\sigma_{\mathrm{S}}^{12} \mathrm{~N}_{1}\right) \mathrm{s}^{ \pm}+i \kappa \mathrm{s}^{\mp}+\tilde{\chi}_{\mathrm{S}}
\end{aligned}
$$

where $\sigma^{12}$ and $\sigma^{21}$ is the absorption and emission cross-section, respectively. $c_{n}$ is the group velocity, $\kappa$ is the coupling coefficient of the propagation fields, $\bar{\alpha}_{P}$ and $\bar{\alpha}_{S}$ is the absorption and scattering loss of the pump and signal fields. The spontaneous emission coupled to the pump and signal is described by the terms $\tilde{\chi}_{\mathrm{p}}$ and $\tilde{\chi}_{s}$, respectively.

In our time domain model, the waveguide laser is divided into a number of equal small sections $M$, as shown in Figure 2, each with length $\Delta z=L / M$, where $L$ is the total length of the waveguide. In each section, the material and structure parameters are assumed to be constant, but they are allowed to change from section to section. By knowing the field value at time $t$ and location $z$, the field for forward (reverse) waves at the next time $t+\Delta t$, where $\Delta t=\Delta z / c_{n}$, can be determined at position $z+\Delta z(z-\Delta z)$ from the equations to be derived below.

The coupled equations in (3) can be solved in time domain by a first-order difference approximation to the partial differential :

$$
\begin{aligned}
& \frac{\partial F^{+}(t, z)}{\partial t}=\frac{F^{+}(t+\Delta t, z)-F^{+}(t, z)}{\Delta t} \\
& \frac{\partial F^{+}(t, z)}{\partial z}=\frac{F^{+}(t, z+\Delta z)-F^{+}(t, z)}{\Delta z} \\
& \frac{\partial F^{-}(t, z)}{\partial t}=\frac{F^{-}(t+\Delta t, z)-F^{-}(t, z)}{\Delta t}
\end{aligned}
$$




$$
\frac{\partial F^{-}(\mathrm{t}, \mathrm{z})}{\partial \mathrm{z}}=\frac{F^{-}(\mathrm{t}, \mathrm{z})-F^{-}(\mathrm{t}, \mathrm{z}-\Delta \mathrm{z})}{\Delta \mathrm{z}}
$$

where $F=p$,s. The evolution of the field $F$ at position $z$ and time $t$ to the adjacent section $z+\Delta z$ at time $t+\Delta t$ can be approximated as:

$$
\begin{aligned}
\mathrm{F}(\mathrm{t}+\Delta \mathrm{t}, \mathrm{z}+\Delta \mathrm{z})-\mathrm{F}(\mathrm{t}, \mathrm{z}) & =\mathrm{F}(\mathrm{t}+\Delta \mathrm{t}, \mathrm{z}+\Delta \mathrm{z})-\mathrm{F}(\mathrm{t}, \mathrm{z}+\Delta \mathrm{z})+\mathrm{F}(\mathrm{t}, \mathrm{z}+\Delta \mathrm{z})-\mathrm{F}(\mathrm{t}, \mathrm{z}) \\
& =\frac{\partial^{2} \mathrm{~F}}{\partial \mathrm{t} \partial \mathrm{z}} \Delta \mathrm{t} \Delta \mathrm{z}+\left.\frac{\partial \mathrm{F}}{\partial \mathrm{t}}\right|_{\mathrm{z}} \Delta \mathrm{t}+\left.\frac{\partial \mathrm{F}}{\partial \mathrm{z}}\right|_{\mathrm{t}} \Delta \mathrm{z}
\end{aligned}
$$

If we choose the relation between the time and spatial steps as $\Delta z=c_{n} \Delta t$ and neglect the second derivative $\partial^{2} F(t$, $z) / \partial t \partial z$, we can obtain the traveling wave formula as follows:

$$
\begin{aligned}
& \mathrm{p}^{ \pm}(\mathrm{t}+\Delta t, \mathrm{z} \pm \Delta z)-\mathrm{p}^{ \pm}(\mathrm{t}, \mathrm{z})=\Delta z\left[\frac{1}{2}\left(-\bar{\alpha}_{\mathrm{P}}+\sigma_{\mathrm{P}}^{21} \mathrm{~N}_{2}-\sigma_{\mathrm{P}}^{12} \mathrm{~N}_{1}\right) \mathrm{p}^{ \pm}(\mathrm{t}, \mathrm{z})+i \kappa \mathrm{p}^{\mp}(\mathrm{t}, \mathrm{z})+\tilde{\chi}_{\mathrm{P}}(\mathrm{z})\right] \\
& s^{ \pm}(\mathrm{t}+\Delta t, \mathrm{z} \pm \Delta z)-s^{ \pm}(\mathrm{t}, \mathrm{z})=\Delta z\left[\frac{1}{2}\left(-\bar{\alpha}_{\mathrm{S}}+\sigma_{\mathrm{S}}^{21} \mathrm{~N}_{2}-\sigma_{\mathrm{S}}^{12} \mathrm{~N}_{1}\right) \mathrm{s}^{ \pm}(\mathrm{t}, \mathrm{z})+i \kappa \mathrm{s}^{\mp}(\mathrm{t}, \mathrm{z})+\tilde{\chi}_{\mathrm{S}}(\mathrm{z})\right]
\end{aligned}
$$

If the number of sections of the laser waveguide $M$ is sufficiently large, the following approximation can be used :

$$
\frac{d N}{d t}=\frac{\Delta N}{\Delta t}
$$

The time dependent rate equation given in (1) can thus be approximated as :

$$
\Delta \mathrm{N}_{2}=\left(\left(\mathrm{R}_{12}+\mathrm{W}_{12}\right) \mathrm{N}_{1}-\left(\mathrm{A}_{21}+\mathrm{R}_{21}+\mathrm{W}_{21}\right) \mathrm{N}_{2}\right) \Delta t
$$

where $\Delta \mathrm{N}_{2}$ is the change of population in the time interval $\Delta \mathrm{t}$. The dynamic behavior of Er-doped waveguide laser can than be solved by using (6) \& (8) in a self-consistent manner. In addition, the implementation of (6) \& (8) is straight forward. The other advantages of this model are the non-linearity such as non-uniform distribution of signal power, pump power as well as $\mathrm{Er}^{3+}$ doped ion can also be taken into consideration.

\subsection{Simulation results}

Figure 3 compares the transient behavior of the waveguide lasers (a) without and (b) with longitudinal grating under a step response of pumped power. As we can see, the grating structure enhances relaxation oscillation frequency. This is because the photon lifetime is reduced significantly by the longitudinal optical feedback. In addition, mode beating is not observed at steady state in Figure $3 \mathrm{~b}$ due to the frequency selective properties of longitudinal grating and single mode operation is maintained at steady state. One fundamental difference between our model and the models given in references $[1,2]$ is that phase relation of the propagation waves is also taken into account in our model such that mode beating phenomena due to the competition of longitudinal modes is observed in our model. In our time domain model, many interesting parameters can be displayed as a function of time as shown in Figure 4.

\section{MODELING OF MODE-LOCKED FIBER LASER}

\subsection{Theoretical model}

The laser structure with a ring configuration used in our analysis is shown in Figure 5. It consists of a single mode polarization-preserving $\mathrm{Er}^{3+}$-doped fiber to provide optical gain for the soliton pulses, an optical isolator to allow unidirectional generation, an amplitude modulator to start the mode-locking regime and a phase modulator to suppress 
Raman self-frequency shift. If the soliton pulses generated from the mode-locked laser have pulse width not less than $100 \mathrm{fs}$, the propagation of soliton pulses can be described by a simplified nonlinear equation [5]:

$$
\frac{\partial u}{\partial z}=j \frac{1}{2} \frac{\partial^{2} u}{\partial T^{2}}+j|u|^{2} u+\frac{1}{2} G u+\frac{1}{2} \gamma_{a} G \frac{\partial u}{\partial T}-j \gamma_{R} u \frac{\partial|u|^{2}}{\partial T}
$$

where $\mathrm{u}$ is the normalized amplitude, $\mathrm{T}$ is the normalized time and $\mathrm{z}$ is the normalized distance. $\gamma_{a}=T_{2}^{i} / \tau_{o}, T_{2}^{i}=1 /\left(\pi \Delta v_{a}\right), \Delta v_{a}$ is the amplification line bandwidth, $\gamma_{\mathrm{R}}=2 \beta \mu \delta, \mu=\left(\mathrm{T}_{\circ} \Omega_{\mathrm{R}}\right)^{-1}, \quad \delta=\left(T_{2}^{R} \Omega_{R}\right)^{-1}, \Omega_{\mathrm{R}}$ is the Raman resonant frequency and $T_{2}^{R}\left(=1 /\left(\pi \Delta v_{R}\right)\right)$ is the characteristic time. In (1), the first term in the right hand side describes the pulse dispersion spreading, the second term describes the Kerr effect, the third and forth terms represent the finite gain bandwidth and pump depletion of $\mathrm{Er}^{3+}$ fiber, respectively. The last term describes the Raman self-frequency shift of the laser. The amplification parameter $G$ is defined as

$$
G=\frac{G_{o}}{\left(1+\sigma W_{l} / W_{p}\right)}
$$

where $W_{l}=\int_{-T / 2}^{T / 2}|u|^{2} d t$ is the laser pulse energy, the coefficient $\sigma$ is proportional to the ratio of pumped and laser transition cross sections, and $W_{p}$ is the pump energy per period.

Equation (9) can be solved by FSAT to analyze the formation of soliton pulses [6]. First, by expressing $\mathrm{u}(\mathrm{z}, \mathrm{T})$ from (9) in terms of Fourier series as shown below:

$$
\begin{aligned}
\sum_{n=-N}^{N} \frac{\partial \hat{u}_{n}(z)}{\partial z} \exp (j n \varepsilon T)= & -\frac{1}{2} j \sum_{n=-N}^{N} n^{2} \varepsilon^{2} \hat{u}_{n}(z) \exp (j n \varepsilon T)+j \sum_{n=-N}^{N} \Psi_{n}(z) \exp (j n \varepsilon T) \\
& -j \gamma_{R} \sum_{n=-N}^{N} \Phi_{n}(z) \exp (j n \varepsilon T)
\end{aligned}
$$

where

$$
\begin{aligned}
& \sum_{n=-N}^{N} \Psi_{n}(z) \exp (j n \varepsilon T)=\left|\sum_{n=-N}^{N} \hat{u}_{n}(z) \exp (j n \varepsilon T)\right|^{2} \cdot \sum_{n=-N}^{N} \hat{u}_{n}(z) \exp (j n \varepsilon T), \\
& \sum_{n=-N}^{N} \Phi_{n}(z) \exp (j n \varepsilon T)=\sum_{n=-N}^{N} \hat{u}_{n}(z) \exp (j n \varepsilon T) \cdot \frac{\partial}{\partial T}\left|\sum_{n=-N}^{N} \hat{u}_{n}(z) \exp (j n \varepsilon T)\right|^{2} .
\end{aligned}
$$

The parameters $\hat{\mathrm{u}}_{\mathrm{n}}(\mathrm{z})$ and $\varepsilon$ are the Fourier amplitude coefficient and the fundamental frequency, respectively. Then, using the orthogonal property of the exponential coefficients, the required $2 \mathrm{~N}+1$ first order partial differential equations can be obtained and are given as follows:

$$
\frac{\partial \hat{u}_{n}(z)}{\partial z}=\underbrace{\left[-j \sigma(n)+\frac{1}{2} G\right] \hat{u}_{n}(z)}_{\text {linear term }}+\underbrace{j \sum_{\forall \mu-v+\lambda=n} \hat{u}_{\mu}(z) \hat{u}_{v}^{*}(z) \hat{u}_{\lambda}(z)+\gamma_{R} \varepsilon \sum_{\forall p+q} q \hat{u}_{p}(z) \Theta_{q}(z)}_{\text {nonlinear term }}
$$

where $\mathrm{n}, \mu, v, \lambda, \mathrm{p}, \mathrm{q}, \mathrm{k}, \mathrm{L}$ and $\mathrm{m}$ are integers of values between $-\mathrm{N}$ and $\mathrm{N} . \quad \sigma(n)=\frac{1}{2} n^{2} \varepsilon^{2}-\frac{1}{2} \gamma_{a} G n \varepsilon$ and $\Theta_{q}(z)=\sum_{\forall a-b=q} \hat{u}_{a}(z) \hat{u}_{b}^{*}(z)$. A time-domain window function term can be introduced into the first order partial differential equations to improve the computational efficiency [7]. The propagation of soliton along the $\mathrm{Er}^{3+}$ doped fiber can be calculated by solving (13) through the Runge-Kutta Method. At each propagation distance, the soliton propagation results of (13) can be transformed to time domain by inverse FFT. 
The transmission function of both amplitude and phase modulators are modeled as shown below:

$$
\begin{aligned}
& \mathrm{K}_{\mathrm{a}}=\mathrm{t}_{\mathrm{a}} \exp \left[-\mathrm{d}_{\mathrm{a}} \sin ^{2}\left(\Omega_{\mathrm{a}} \mathrm{T}+\phi_{\mathrm{a}}\right)\right] \\
& \mathrm{K}_{\mathrm{p}}=\exp \left[j \mathrm{~d}_{\mathrm{p}} \cos \left(\Omega_{\mathrm{p}} \mathrm{T}+\phi_{\mathrm{p}}\right)\right]
\end{aligned}
$$

where $\mathrm{d}_{\mathrm{a}}\left(\mathrm{d}_{\mathrm{p}}\right), \Omega_{\mathrm{a}}\left(\Omega_{\mathrm{p}}\right)$ and $\phi_{\mathrm{a}}\left(\phi_{\mathrm{p}}\right)$ are the depth, frequency and phase of the amplitude (phase) modulation, respectively. The information of soliton pulse after the gain section in time domain is used as the initial boundary conditions for the amplitude and phase modulators. The output of the modulators will then be transformed back into frequency domain as the initial conditions for the $\mathrm{Er}^{3+}$ doped fiber. The above process will repeat until the predefined propagation distance (i.e. number of pass) is reached.

\subsection{Simulation results}

Figures 6 and 7 shows the evolution of soliton pulses in time and frequency domains generated from pure active mode-locking and with self phase modulation respectively. In the calculation, the initial pulse shape is assumed as $u(0, T)=\operatorname{sech}(T)$. The other parameters used in the calculation are the same as [5] except $K=5$ and $T_{0}=8$.

Figure 8 shows the vraiation of pulse with gain parameter $G_{o}$. It is observed that the pulse width is decreased exponentially with $G_{\circ}$ and the profile is close to that given in [5]. Therefore, we have shown that the simulation results are well agreed with [5] and the proposed FSAT method in modeling of active model-lock fiber laser is reliable. The influence of energy loss coefficient, pump energy and active fiber length on pulse power is shown in Figure 9.

\section{CONCLUSIONS}

The paper has presented two numerical simulation models to illustrate the usefulness of simulation as a modeling and teaching tool to enhance the understanding of optical devices and systems. Development of the simulation programs can serve as student research projects. The simulation programs developed can serve as teaching tools or as project assignments to illustrate the performances of the waveguide laser and fiber laser as a function of device parameters.

\section{REFERENCES}

[1] J. Ohya, H. Sato, M. Mitsuda, T. Uno \& T. Fujta, J. Lightwave Technol., vol. 13, no.11, pp.2129-2135, 1995.

[2] M. Dinand and Ch. Schutte, J. Lightwave Technol., vol. 13, no. 1, pp. 14-23, 1995.

[3] P. W. Milonni and J. H. Eberly, Laser. New York: Wiley, 1988, ch. 10.

[4] A. E. Siegman, Lasers. Mill Vally, CA: Univ. Science Books, 1986, ch. 13.

[5] E. Marti-Panameno, J. J. Sanchez-Mondragon and V. A. Vysloukh, IEEE Journal of Quantum Electronics, Vol.30, No.3, pp.822-826, 1994.

[6] H.Ghafouri-Shiraz, P. Shum and M. Nagata, IEEE J.Quantum Electron., vol.31, no.1, pp. 190-200, 1995.

[7] P. Shum and S. F. Yu, IEEE Photonics Technology Letters, Vol. 8, no. 10, pp.1364-1366, 1996. 


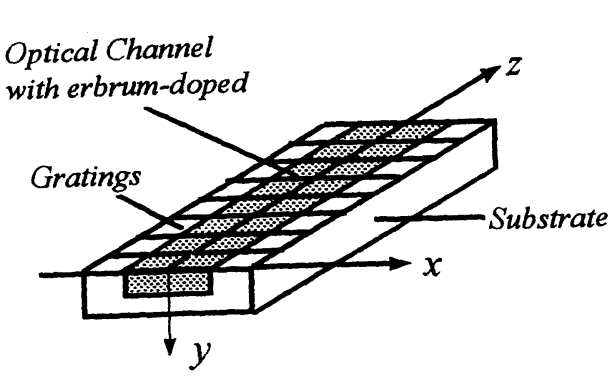

Figure 1. Schematic of $\mathrm{Er}^{3+}$-doped waveguide laser

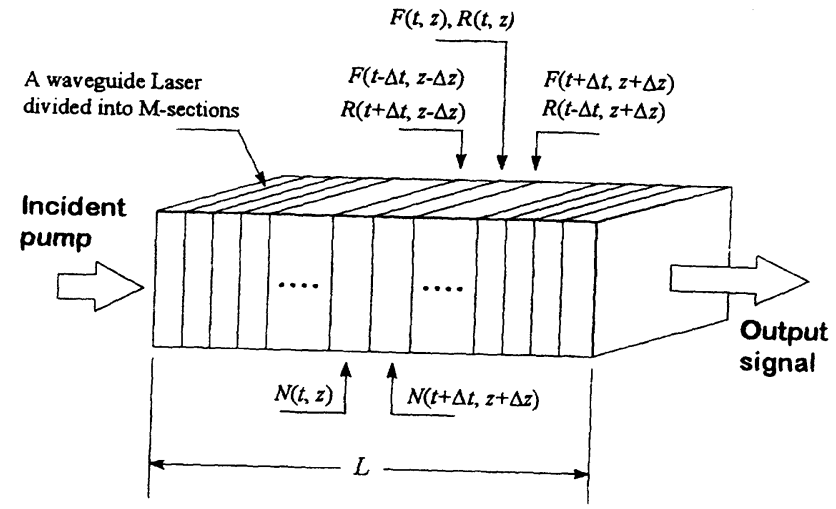

Figure 2. A waveguide model showing the time-domain algorithm principle.

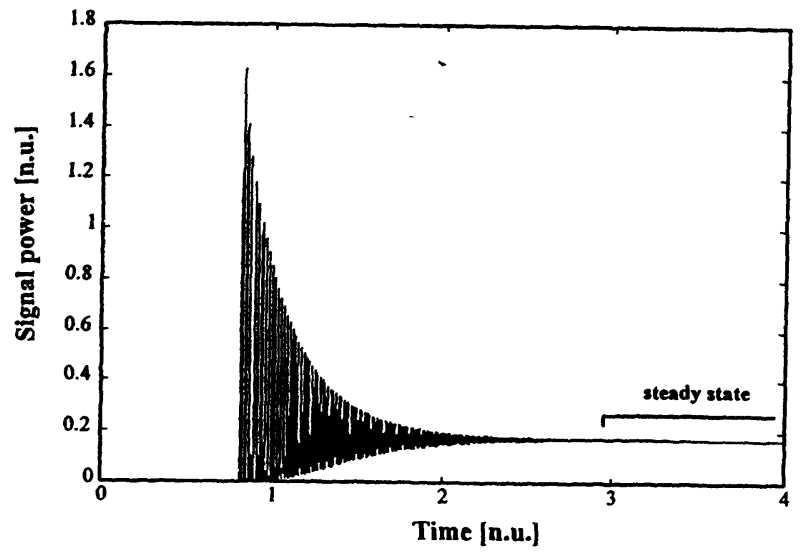

Figure 3. Calculated relaxation oscillations of the Er-doped waveguide laser (a) without grating, (b) with grating under a step injection optical power.
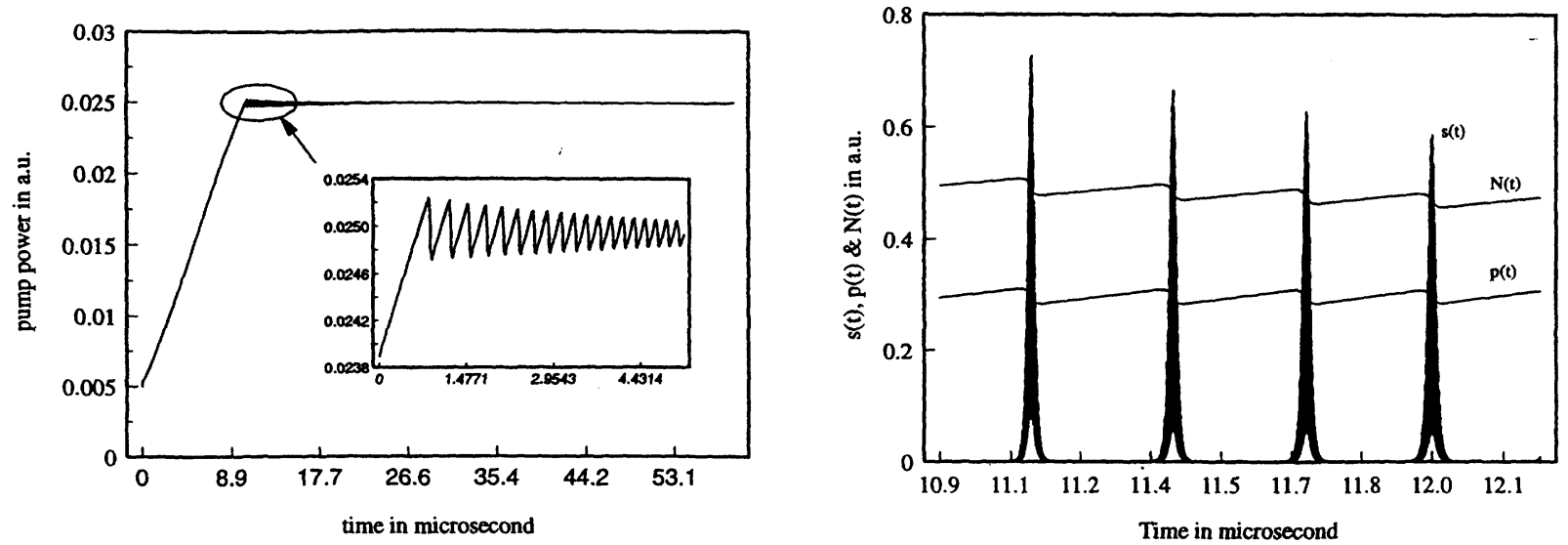

Figure 4. Time evolution of (a) the pump power, (b) expanded view of the inversion population, signal and pump power. 


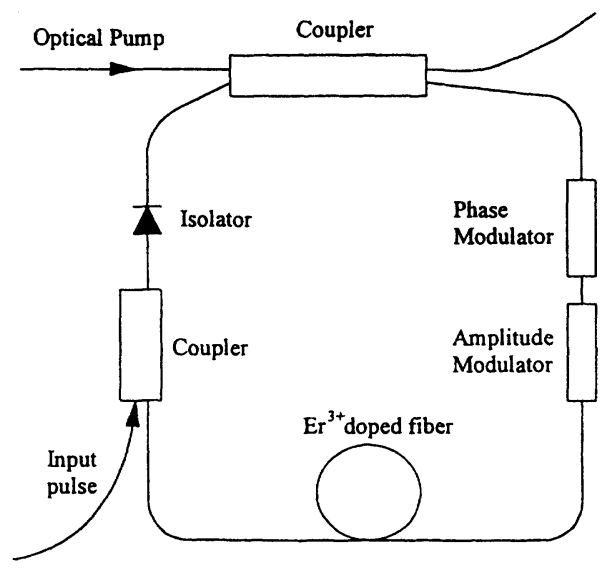

Figure 5. The schematic of an active mode-lock $\mathrm{Er}^{3+}$ doped fiber laser in ring configuration.
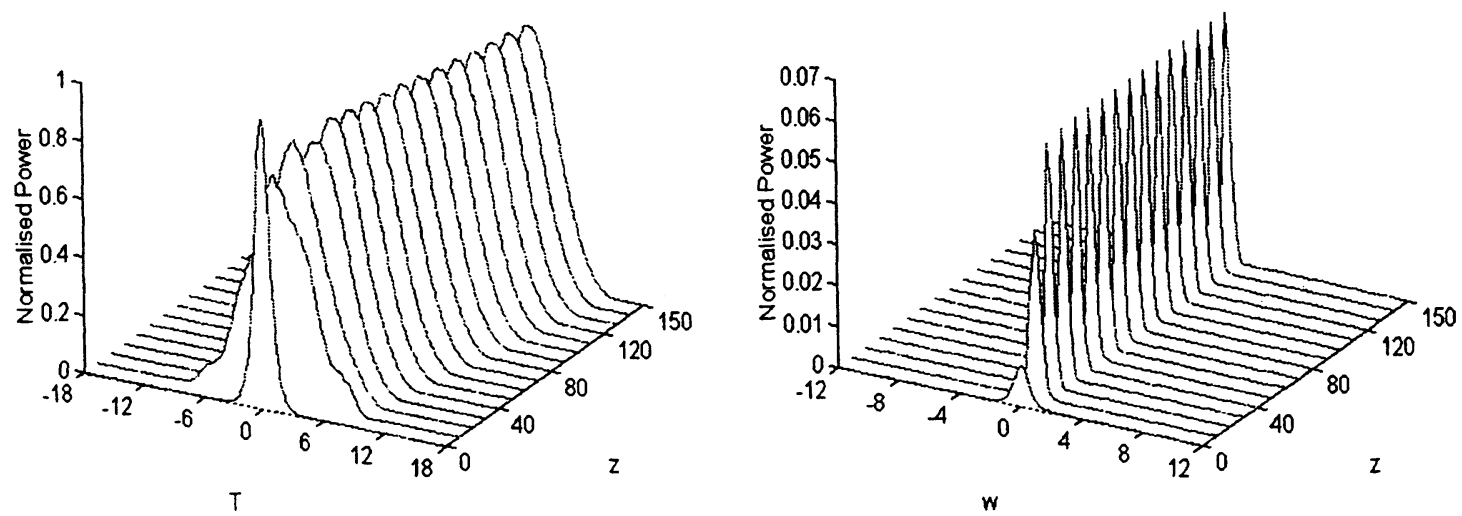

Figure 6. The propagation of soliton pulses in an pure active mode-locked fiber laser (without self-phase modulation).
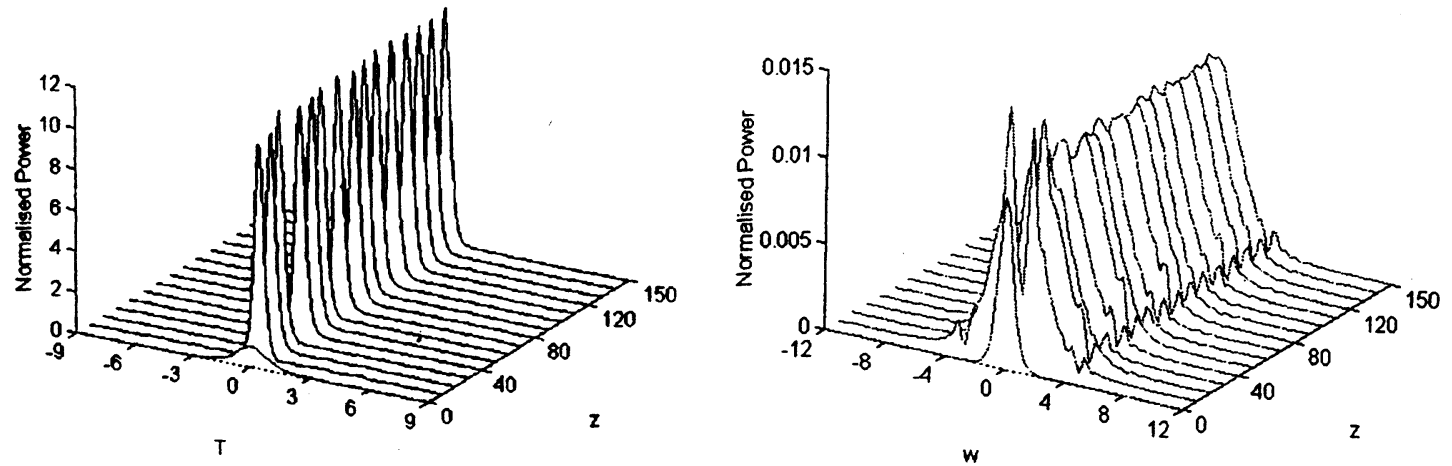

Figure 7. Evolution of laser radiation in the case of soliton shaping. 


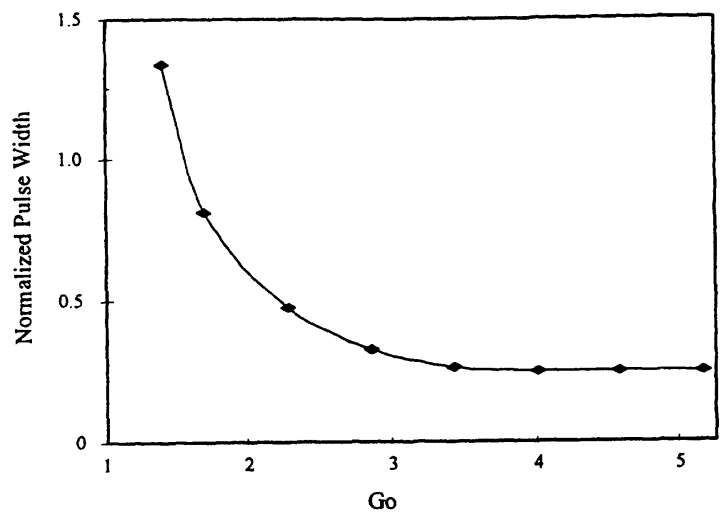

Figure 8. Plot of steady state pulse width against gain parameter $\mathrm{G}_{0}$.
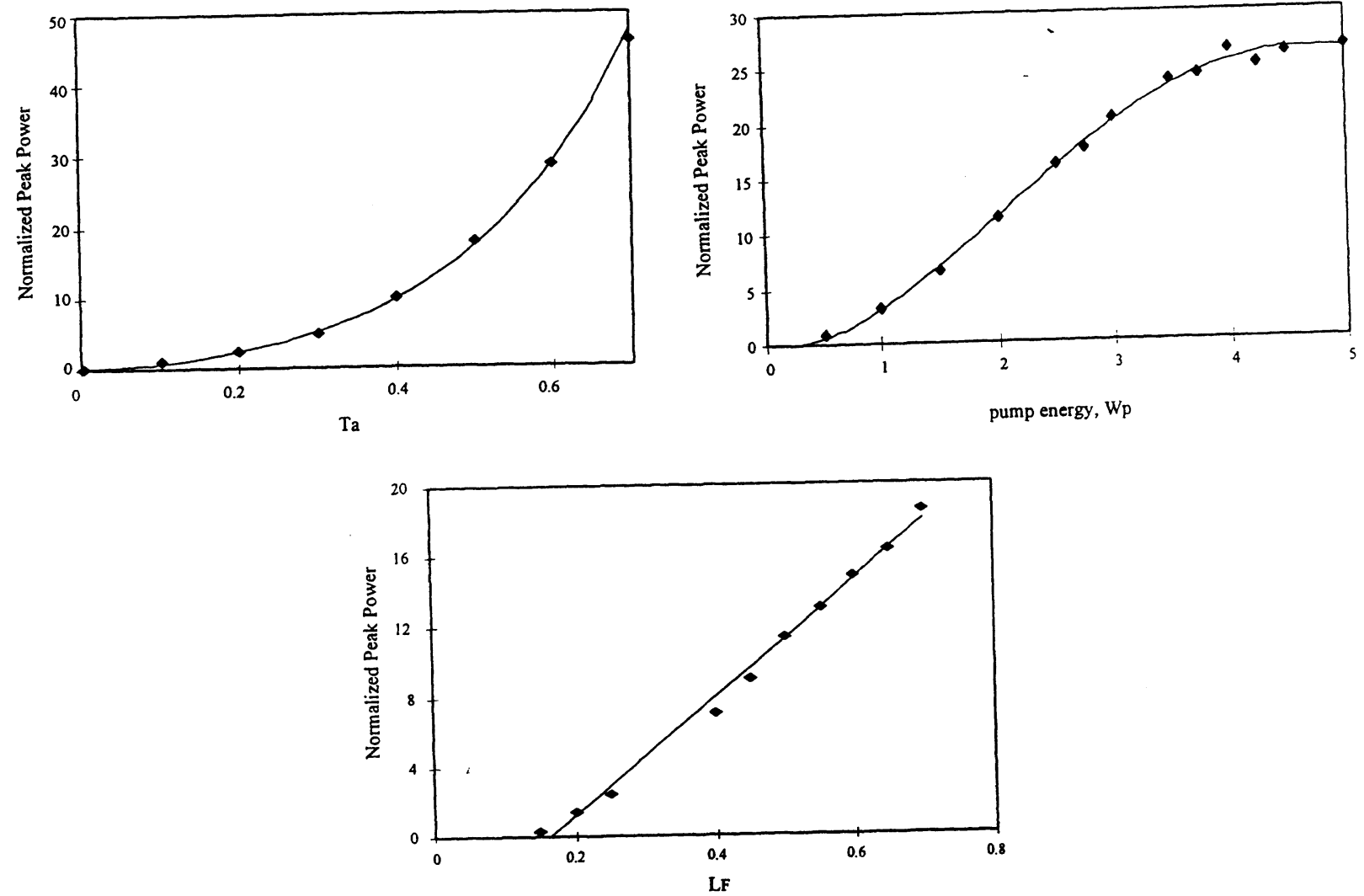

Figure 9. Plot of steady state peak power against (a) energy loss coefficient of amplitude modulator, (b)pump energy, and (c) length of active Er-doped fiber. 